chief executive officer Niels Fertig. It is the only automated device that addresses the need for low throughput with high accuracy, Fertig claims.

The Port-a-Patch system uses planar borosilicate glass chips $(100-\mu \mathrm{m}$ thick) in which a conical pore of $1-\mu \mathrm{m}$ diameter is micromachined. The pore has the threedimensional geometry of an inverted pipette tip and cells are simply positioned via suction. It creates a strong electrical seal with the cell and is ideal for whole-cell patch clamping. Single-channel recordings can be performed in a cell-attached configuration. A softwarecontrolled eight-channel microfluidics add-on can deliver sufficiently rapid changeover of solutions to allow the study of ligand-gated channels. A robotic version of the system that will run 16 patches at a time is in prospect.

The Flyscreen, an alternative approach for moderate-throughput applications from flyion of Tübingen, Germany, can perform 100 to 500 independent whole-cell screens per day. The instrument uses glass micropipettes, into which cells are loaded. As the cells settle, a single one falls towards the tip and wedges near the opening. Carefully controlled suction draws the cell into a tight fit and further trains of pressure pulses disrupt the membrane, leaving a patch of cell membrane spanning the pipette's lumen. A plastic jacket moulded around the pipette enables robotic handling. The machine holds up to six pipettes and each channel runs independently, so pipettes can be discarded as soon as the cell fails.

${ }^{\alpha}$ Glass blowing enables us to be flexible in the shape and geometry of the tips", says inventor Albrecht LeppleWienhues, founder and chief executive officer of flyion. This allows tailoring to suit different types of cells, and the new Flip-the-tip Large tips, which have a bowl at the base, enable the machine to monitor ligand-gated channels. ${ }^{\alpha}$ The bowl at the base gives us enough space to intro-duce a 130 $\mu \mathrm{m}$ diameter quartz needle," says Lepple-Wienhues. In flyion's standard tips, solution exchange takes about 60 seconds, but the new tips allow solutions to be puffed directly on to the cell through the quartz needle and give exchange rates of less than 50 milliseconds, while continuous recording is being carried out from each cell.

\section{Patch-clamp economics}

A report published in September by the Cambridge-based market-research consultancy HTStec makes interesting reading for those involved in the ion-channel industry. According to HTStec, the pharmaceutical and biotech market will spend around $\$ 32$ million in 2005 on automated patch-clamping machines. "We predict that sales will peak in 2006 at around 200 units a year," says HTStec director John Comley. In addition to this, the report estimates that for automated patchclamping, labs spend around $\$ 10$ per data point for safety assessment and $\$ 3.00$ per data point in primary screening. Companies claim they would be more comfortable paying around $\$ 6$ and $\$ 0.60$ respectively. HTStec's survey of 66 companies and universities indicated that manual patch damping was still the preferred option in assay development and safety assays such as hERG compliance, but automated patch damping was the method of choice for secondary screening, lead optimization and early noncompliant hERG liability testing.

The highest possible throughput of some 3,000 data points a day is still far short of the 20,000 data points that respondents said they would like to get from a machine. Many were looking forward to machines that measure ligand-gated channels much more cheaply. As these account for $29 \%$ of all ion channels studied, this is a potentially big market.

With genomics and proteomics creating a resurgence in cellular and systems research, there is every reason to believe that ionchannel research will become even more important in the coming decade.

Pete Moore is a science writer based near Bristol, UK.

Sigworth Laboratory

info.med.yale.edu/cmphysiol/sigworth/ HTStec

www.htstec.com

\title{
SIGNALS OF DISEASE
}

Protein kinases are linked to numerous disease states, including cancer, arthritis, diabetes, cardiovas cular diseases and neurologicaldisorders. Gleevec from Novartis was the first compound active against a kinase (the Abl kinase) to be approved as a treatment - for certain gastrointes tinal tumours and chronic myeloid leukaemia.

The market for kinases is large. "More than $\mathbf{2 5 \%}$ of new drugs being developed today are based on kinase technology," says Jeff Linton, president of Upstate of Charlottesville, Virginia, which offers one of the largest collections of kinases. A flagship of Upstate's operation is its KinaseProfiler service, run from Dundee in Scotland. This provides quantitative characterization of compounds ag ainst an everexpanding panel of human protein kinases in adirect radiometric assay. The panel currently

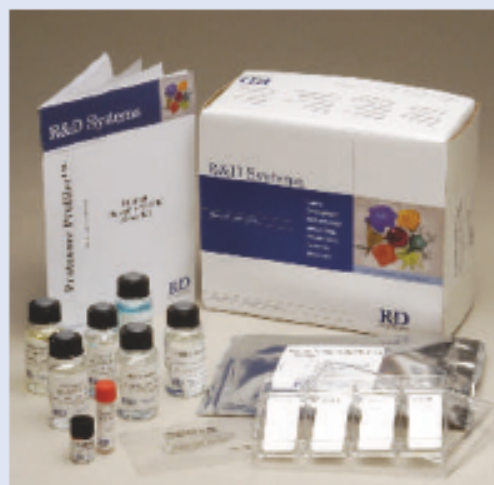

R\&D Systems' Phos pho-MAPKarray tracks phosphorylated kinases.

contains around 230 kinases, almost $50 \%$ of the total number of human kinases in the genome. A new focus for Upstate is the addition of naturally occurring mutant kinases as they are identified.

Attention is also focusing on the newly emerging Gleevec-resistant mutants of $\mathrm{Abl}$, and mutant forms of other kinases including the epithelial growth factor (EGF) receptor, as these mutations can alter an inhibitor's efficacy. One of these mutations involves a single 'gat ekeeper' amino acid. Mutations in this amino acid can prevent therapeutic compounds from binding effectively without affecting the enzyme's activity. "The search is on for successful inhibitors that are not sensitive to changes at the gatekeeper site," says Steve Davies, director of Upstate's drug discovery segment.

Upstate is helping this search by adding eight different mutant kinases to their portfolio, including ones for Kit, EGFR, A bl, Flt3 and p38/SAPK2a - and, says Davies, there aremore in the pipeline.

A highly specific set of antikinase antibodies makes up R\&D Systems' Proteome Profiler
Phospho-MAPK Array. This allows analysis of the phosphorylation status of 19 key signalling proteins, including members of all three major families of mitogenactivated protein kinases - the extracellular signal-regulated kinases, c-Jun $\mathrm{N}$-terminal kinases, and the 38 kinases. These enzymes play essential roles in numerous signalling pathways that underlie cell function and disease.

Signalling pathway analysis products from Beckman Coulter of Fullerton, California, are also devoted to looking at intracellular activated (phosphorylated) kinases. One strength is that these reagents can be used on many different types of specimen including whole blood, and can resolve activated and inactivated kinases in whole blood cells, according to Michel Herbert, marketing manager for Beckman Coulter. 